\title{
Implementare la tecnica del buttonhole: un'esperienza monocentrica
}

\author{
Andrea Novelli, Sofia Amabili, Beatrice Cicconi, Luca Mandolesi, Giuseppe Solano, Elena Oliva, Francesco Bruni \\ ASUR Marche, UOC Nefrologia e Dialisi, Area Vasta N. 5, PO Ospedale "Madonna del Soccorso", San Benedetto del Tronto (AP)
}

\begin{abstract}
How to use the buttonhole technique: a single-center experience
Suitable vascular access is a prerequisite for adequate and efficient hemodialysis. Cannulation of an arteriovenous fistula (AVF) can be difficult in some patients, and this has led to the widespread use of the buttonhole (BH) technique. This paper describes our experience in $\mathrm{BH}$ technique practice and provides a pocket guide for clinicians and nurses who would like to approach it. Our experience shows that inadequate technique management is often associated with a higher vascular access infection rate. We used the BH technique in 25,985 punctures, recording only 13 puncture site infections. Two AVFs were lost due to severe infection and symptomatic arterial hypotension. The inclusion criteria were widened in order to collect data from most of the hemodialysis population. Our results confirm the validity of the $\mathrm{BH}$ technique and will serve to make crucial clinical considerations as well as strategies to prevent $\mathrm{BH}$ technique-related complications.
\end{abstract}

Keywords: Buttonhole, Complications, Hemodialysis, Vascular access

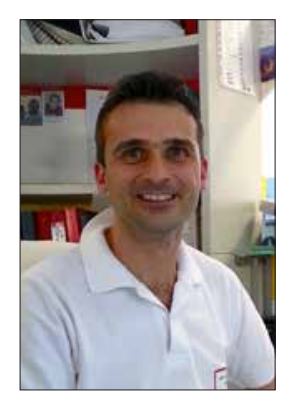

Andrea Novelli

\section{Introduzione}

La fistola artero-venosa (FAV) è considerata l'accesso vascolare più efficace e duraturo per poter effettuare sedute emodialitiche adeguate ed efficienti nel paziente nefropatico cronico (1). Inoltre l'accesso vascolare nativo è legato a un minor numero di episodi infettivi (2).

Negli anni diverse sono state le tecniche di venipuntura proposte. Le più diffuse sono quella ad area e quella $a$ scala di corda. La prima spesso complicata dallo sviluppo di dilatazioni aneurismatiche nell'area di puntura. La seconda, la tecnica a scala di corda, è purtroppo spesso difficilmente praticabile nei pazienti con breve tratto vascolare pungibile o per la presenza di zone aneurismatiche o di cute atrofica.

Queste criticità, assieme al dolore spesso lamentato dai pazienti all'atto dell'incannulazione, ci hanno indotto

Accepted: November 3, 2016

Published online: June 5, 2017

Indirizzo per la corrispondenza:

Dr. Andrea Novelli

Ospedale "Madonna del Soccorso"

Via Luciano Manara 7

62074 San Benedetto del Tronto (AP)

parnan75@gmail.com ad adottare la tecnica di puntura a occhiello o buttonhole $(\mathrm{BH})$ in un largo numero di pazienti afferenti al nostro Centro.

Diversi studi riportati in letteratura hanno valutato i pro e i contro di questa tecnica di venipuntura. La tecnica del $\mathrm{BH}$ determina una significativa riduzione delle complicanze quali dilatazioni aneurismatiche, dolore al momento della puntura, tempo di emostasi, errori di incannulazione con conseguente riduzione di ematomi e pseudoaneurismi, e inoltre preserva l'estetica dell'arto, cosa molto cara al paziente $(3,4)$. Dati recenti dimostrano inoltre un più basso numero di interventi di angioplastica nei pazienti che utilizzano la tecnica $\mathrm{BH}$. Viceversa a sfavore di questa tecnica si è osservato una maggiore incidenza di infezioni (5).

Questo lavoro vuole proprio far conoscere la nostra esperienza, i risultati ottenuti in termini di efficienza dialitica e sopravvivenza della fistola e l'uniformità dell'addestramento di tutto il personale infermieristico.

\section{Pazienti e Metodi}

Da marzo 2010 a marzo 2013 sono stati arruolati nello studio 57 pazienti che rispettavano i seguenti criteri di inclusione:

- $\quad$ presenza di una fistola nativa;

- difficoltà di incannulamento per ridotto tratto venoso disponibile;

- incannulazione dolorosa. 
Sono stati esclusi i pazienti portatori di fistola protesica e catetere venoso centrale (CVC). Sono stati pertanto valutati 83 pazienti dei quali 26 sono stati esclusi perché portatori di CVC permanente e impossibilitati al confezionamento di FAV. L'auto-incannulazione è stata eseguita in un solo caso.

La tecnica di puntura utilizzata è quella descritta da Twardowski e Kubara nel 1979 (6).

Tutto il personale infermieristico è stato addestrato alla metodica dell'incannulamento con $\mathrm{BH}$. La parte iniziale della creazione del tunnel è stata monitorata con supporto fotografico, video e cartaceo. Sono state descritte dettagliatamente l'inclinazione e la direzione dell'ago tagliente usato per formare il tunnel al fine di poter replicare la tecnica a ogni venipuntura successiva, anche da operatori diversi.

La tecnica originale della puntura a sito costante prevede che il tunnel cicatriziale formato per venipuntura sempre nella stessa e precisa sede sia eseguito dallo stesso operatore affinché si pratichi la puntura con la medesima angolazione. Un tunnel ben confezionato, infatti, permetterà di utilizzare successivamente l'ago smusso agevolmente per mano di operatori anche diversi. Viceversa la complicanza più nota di un tunnel cicatriziale non ben formato è quella del cosiddetto "effetto rimbalzo o trampolino", che si verifica quando l'ago smusso introdotto nel tunnel cicatriziale non entra nel vaso perché non trova corrispondenza con la breccia cicatriziale che si viene a formare sulla parete del vaso.

La nostra esperienza mostra che "I'effetto rimbalzo", si instaura sia che il tunnel cicatriziale venga formato da un solo operatore che da operatori diversi. Tale problematica si può verificare anche in FAV con tunnel ben formato e anche dopo un lungo periodo di uso degli aghi smussi senza alcuna difficoltà. In questi casi si è deciso di utilizzare di nuovo per sei sedute l'ago tagliente, sempre nello stesso sito, con la solita tecnica. Quando questa strategia è risultata inefficace abbiamo proceduto al confezionamento di un nuovo tunnel in altro sito

Altre argomentazioni inerenti alla tecnica del BH su cui

\section{SCHEDA MONITORAGGIO FAV}

PAZIENTE: MESE:

ANNO: CONFEZ. IL

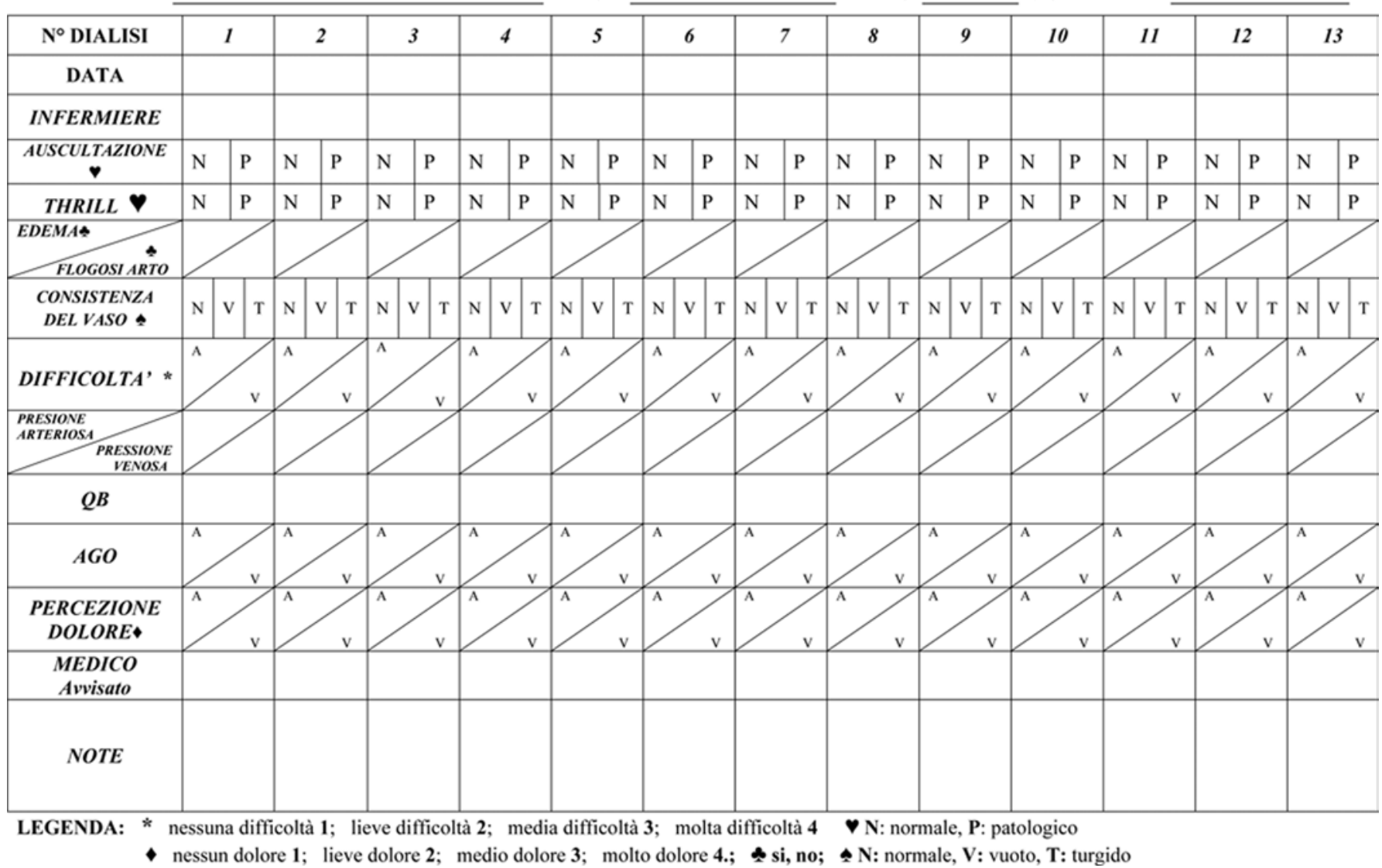

Scheda 1 - Scheda monitoraggio FAV. 
cercare di gettar luce sono la riduzione del dolore, la minore difficoltà di incannulazione da parte del personale, e l'aumentata incidenza di infezioni dell'accesso vascolare (7).

II dolore e la difficoltà di incannulamento sono stati classificati attraverso una scheda di autovalutazione (Scheda 1) articolata in 4 punti da un minimo di 1 , in assenza di dolore o difficoltà, a un massimo di 4 , in caso di forte dolore o di alta difficoltà. L'incidenza delle infezioni dei siti di venipuntura o sistemiche, è stata valutata mediante osservazione e registrazione dei parametri clinici di infezione ed esecuzione di esami colturali con tampone e/o emocolture ove sospetta la presenza.

\section{Creazione del tunnel}

Come già specificato precedentemente, anche se la tecnica prevede che il tunnel venga formato da un singolo infermiere di riferimento, non essendo ciò sempre possibile, abbiamo deciso che anche le iniziali inserzioni dell'ago tagliente venissero praticate da diversi operatori, cercando ovviamente di limitare il numero di operatori nella fase di formazione del tunnel (massimo tre operatori).

La cartella di ogni paziente è stata dotata di una foto della FAV al momento dell'incannulamento per mostrare la direzione corretta dell'ago nel tunnel (formato A4, stampa di file jpeg), mentre per l'inclinazione dell'ago ci siamo dotati di supporto video riproducibile sui terminali presenti in sala dialisi. L'ago tagliente, grazie al supporto foto-video, viene introdotto in modo tradizionale afferrandolo per le alette e seguendo il più possibile l'angolazione della puntura precedente. Dopo un numero di punture con ago tagliente, che varia da paziente a paziente per ottenere la formazione del tunnel cicatriziale, si passa all'uso dell'ago smusso che viene introdotto con la tecnica "touch cannulation" (Fig. 1), cioè inserendo l'ago smusso delicatamente, afferrandolo per il tubicino, con una leggera pressione e

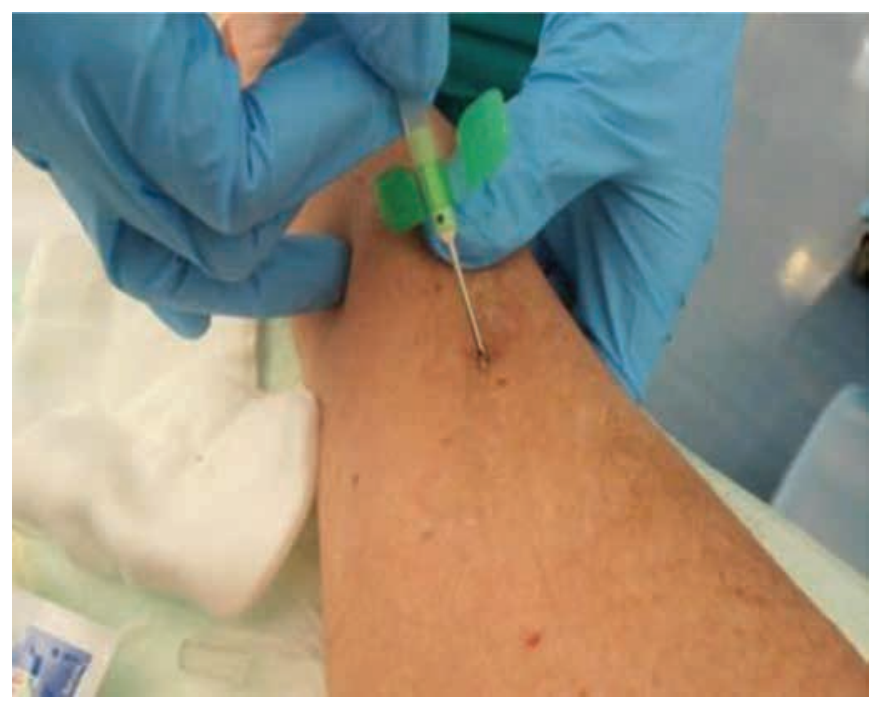

Fig. 1 - Tecnica "touch cannulation".

rotazione, basculando a destra e a sinistra per più volte, fino a penetrare all'interno del vaso.

\section{Risultati}

In 36 mesi sono stati arruolati 57 pazienti afferenti al nostro Centro dialisi. Di questi 57 pazienti, 35 erano uomini e 22 donne, 46 pazienti prevalenti (3 diabetici) e 11 incidenti. Tutti erano dializzati con un ritmo trisettimanale (in media 4 ore/sessione); il 30\% dei pazienti è stato trattato con modalità HDF-on line e con filtro ad alto flusso, con flusso sangue medio di $300 \mathrm{~mL} / \mathrm{min}$.

L'accesso vascolare prima di passare all'utilizzo dell'ago smusso in media è stato punto con ago tagliente per 13 volte.

Nei 36 mesi di osservazione sono state praticate 25.985

TABELLA I - Risultati ottenuti dal nostro Centro con la tecnica BH

\begin{tabular}{|c|c|c|c|c|}
\hline Nr. Pazienti & Tempo di Osservazione (mesi) & Infissioni Totali & Infissioni Ago smusso & Difficoltà Incannulamento \\
\hline \multirow[t]{3}{*}{57} & 36 & 25985 & 21.559 & Nessuna $92,4 \%$ \\
\hline & & & & Lieve $5,3 \%$ \\
\hline & & & & Alta $0,8 \%$ \\
\hline
\end{tabular}

\begin{tabular}{lcccc}
\hline Nr. Ematomi & Nr. Pazienti intolleranti al disinfettante & Nr. FAV Infette & Nr. FAV Chiuse & Percezione del Dolore \\
\hline $47^{*}$ & $11^{* *}$ & 13 & $2 * * *$ & Nessuna $95,2 \%$ \\
& & & Lieve 4,0\% & Moderata 0,6\% \\
& & & Alta 0,2\% \\
\hline
\end{tabular}

\footnotetext{
* tutti causati da ago tagliente.

** di cui 8 trattati con la sola sostituzione della clorexidina $2 \%$.

***1 per infezione severa e 1 per ipotensione.
} 


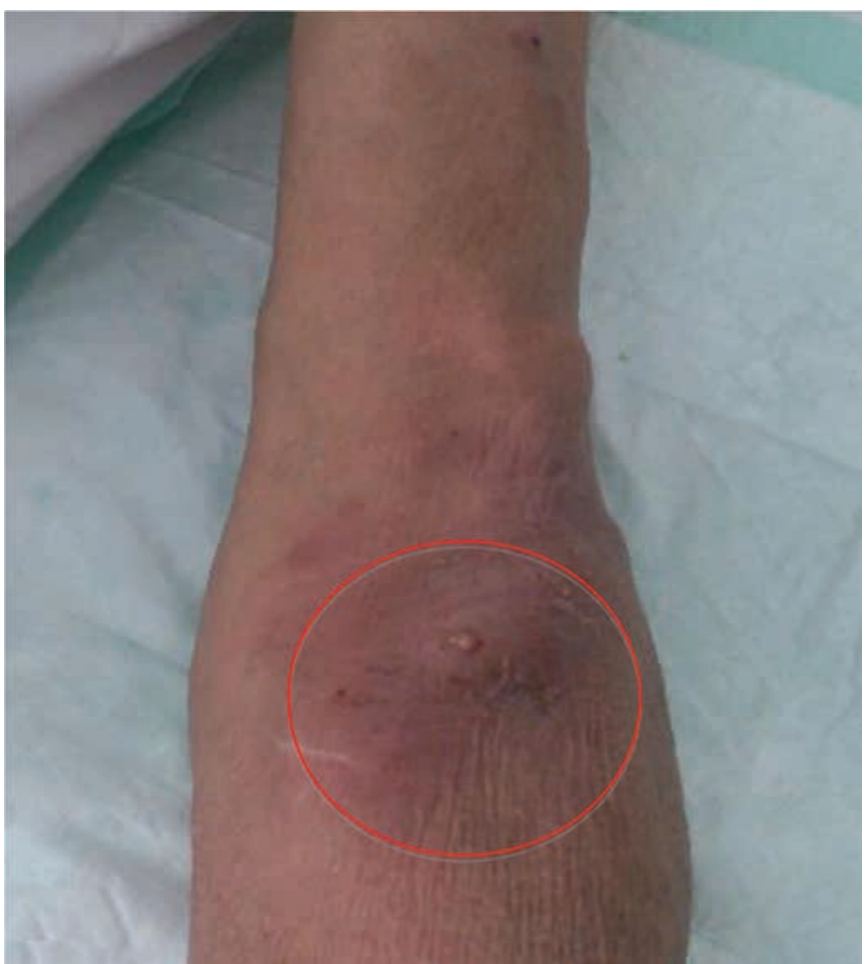

Fig. 2 - Quadro ispettivo nel caso di infezione di un sito di puntura con tampone positivo per Staphylococcus Aureus.

infissioni dell'ago con tecnica di venipuntura BH (Tab. I). 8 pazienti ( $14 \%$ del totale) hanno avuto 13 infezioni del sito di puntura accertate mediante esame colturale da tampone: nel nostro Centro, pertanto, a ogni infissione dell'ago la probabilità di causare un'infezione è stata dello $0,05 \%$. II 77\% delle infezioni (10 infezioni) si sono verificate nei primi 14 mesi. Gli esami colturali hanno evidenziato i seguenti germi: Staphylococcus Aureus ed Epidermidis (Fig. 2). Tali infezioni sono state trattate con disinfettanti locali e antibiotico per os.

Due accessi vascolari sono stati persi: uno per grave infezione con batteriemia (emocoltura positiva per S. Aureus) e l'altro per ipotensione severa. Nel primo caso si trattava di paziente affetto da Lupus Eritematosus Sistemico (LES), in trattamento steroideo ad alte dosi. A fronte del grado di immunodepressione si è deciso pertanto di aggiungere ai criteri di esclusione dalla puntura $\mathrm{BH}$ anche l'immunodepressione importante.

L'uso prolungato di soluzione disinfettante su base alcolica (clorexidina alcolica al $2 \%$ ) ha determinato sensibilizzazione della cute in 11 pazienti (19\% del totale) (Fig. 3); in 8 pazienti è stato sufficiente cambiare disinfettante (lodopovidone su base acquosa 7,5\%) per ripristinare il normale trofismo cutaneo. Negli altri 3 pazienti si è deciso di non utilizzare il sito di puntura interessato dalla dermatite e applicare corticosteroidi per uso topico per la sola durata della seduta di dialisi, ottenendo la risoluzione del problema dopo quattro applicazioni e recuperando così il sito.

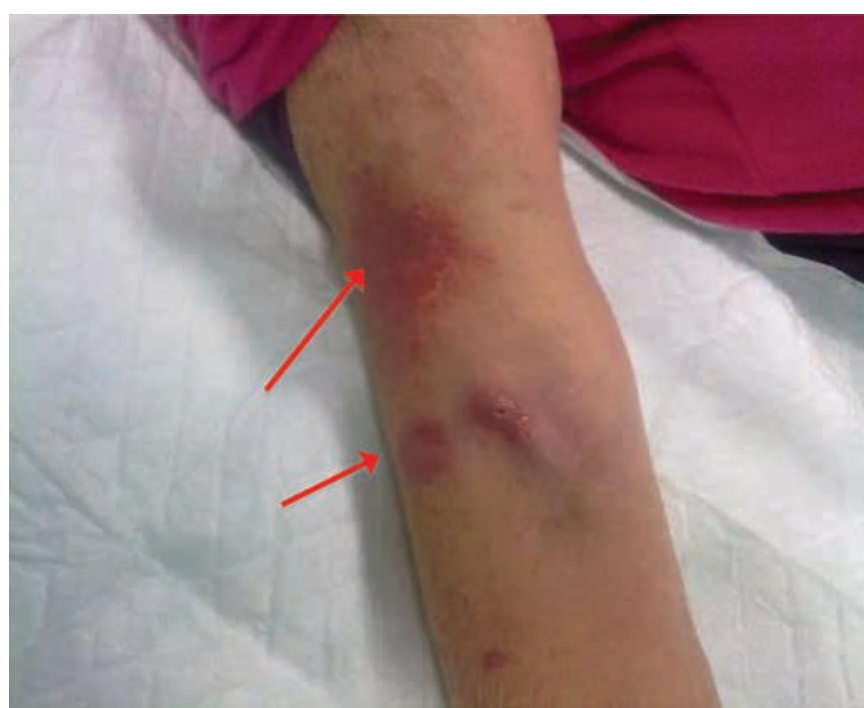

Fig. 3 - Dermatite da contatto nei siti di venipuntura.

Durante il periodo di studio, all'esame obiettivo non si sono osservati aneurismi di nuova insorgenza o peggioramento di quelli noti.

In 2 pazienti, entrambi affetti da diabete mellito, è occorsa una trombosi parziale del tratto venoso vicino al sito di puntura, in entrambi interessando un solo sito di punzione (in tutti i casi trattasi del sito arterioso). In uno dei 2 casi ciò ha implicato l'abbandono del sito (per apposizione trombotica), mentre nell'altro abbiamo utilizzato la puntura monoago per un mese somministrando eparina a basso peso molecolare con successo e permettendoci di riutilizzare lo stesso sito.

Grazie alla raccolta dei dati mediante una scheda di monitoraggio FAV (Scheda 1, Tab. I), sappiamo che il 92,4\% degli infermieri non ha avuto difficoltà nell'inserimento degli aghi in accordo con altri autori (7), e che durante l'incannulazione con ago smusso il 95,2\% dei pazienti non ha avuto dolore, risultati anche in questo caso conformi con quanto riportato in letteratura (8-11).

Anche per quanto riguarda il tempo di emostasi, la riduzione ottenuta con la tecnica BH rispetto all'utilizzo dell'ago tagliente è comparabile con quanto descritto in letteratura (8-11).

\section{Discussione}

L'utilizzo della tecnica BH per I'incannulazione della FAV è in crescita in tutto il mondo. I benefici clinici però possono essere oscurati da un aumento delle complicanze infettive, per questo è di vitale importanza la stretta osservanza di appropriate tecniche di disinfezione al momento dell'attacco.

La nostra esperienza, seppur con i limiti di uno studio monocentrico, ha dato risultati molto positivi, sia per l'interesse dimostrato da tutto il personale sanitario sia per i risultati ottenuti in termini di basso numero di infezioni e 
di sopravvivenza dell'accesso.

Da questa nostra esperienza abbiamo compreso che le infezioni riscontrate nei nostri pazienti sono imputabili essenzialmente a una inadeguata applicazione delle manovre di disinfezione e/o a una non corretta rimozione della crosta prima della venipuntura. Infatti, il personale dopo aver frequentato seminari di aggiornamento interni ed esterni, ha al fine stilato una serie di protocolli per l'utilizzo della tecnica, riguardanti in particolar modo l'igiene del braccio della FAV, la disinfezione prima dell'incannulazione e la corretta modalità di rimozione delle croste. Tale protocollo di disinfezione standardizzato e riportato nell'Appendice 1, viene oggi utilizzato da tutti gli operatori al fine di evitare le complicanze infettive ampiamente descritte $(12,13)$. A parer nostro comunque ciò deve essere accompagnato da un training iniziale di tutto il personale ma soprattutto da un programma di aggiornamento continuo per ovviare alla possibile perdita di rigore nell'esecuzione della metodica prestabilita.

L'altra problematica indelebilmente legata alla tecnica $\mathrm{BH}$ è quella organizzativa, ovvero la necessità di avere un infermiere di riferimento dedicato alla formazione del tunnel cicatriziale per oltre sei dialisi consecutive. Tale criticità è stata da noi superata soprattutto mediante l'uso di materiale fotografico e video di supporto (14), evitando pertanto anche la possibile insorgenza di dipendenza psicologica del paziente a un solo operatore riuscendo così a mantenere la massima "tutti utili ma nessuno indispensabile".

I protocolli stilati e riportati in Appendice 1 sono stati sempre condivisi e applicati da tutti senza alcuna personalizzazione. Come facilmente comprensibile il fattore determinate è comunque stato quello di avere un team collaborativo e con ottime relazioni tra tutte le figure professionali coinvolte compresi i pazienti.

\section{Conclusioni}

Negli ultimi trent'anni abbiamo assistito a un importante sviluppo nelle tecniche emodialitiche con significative innovazioni. Ciò non è stato altrettanto evidente per gli accessi vascolari, che rappresentano notoriamente e da molti anni un punto critico per la salute dei pazienti in dialisi (15).

La FAV nativa rimane l'accesso vascolare ideale (migliore durata, minori interventi, migliori prestazioni), ma la sua efficienza può essere ridotta da incongrue metodiche di venipuntura. Parte della letteratura scientifica conclude in merito che la tecnica $\mathrm{BH}$ può permettere di ridurre alcune delle più frequenti complicanze dell'uso della FAV (errori di incannulazione, formazione di aneurismi, ematomi e punture dolorose). I contro a tale tecnica sono soprattutto legati all'elevato rischio infettivo.

In conclusione la nostra esperienza ci permette di affermare che la tecnica $\mathrm{BH}$ è valida, può permettere di prevenire le tipiche complicanze dovute all'uso delle FAV, e che anche le complicanze infettive possono essere contenute in modo assai efficace. Inoltre, possiamo affermare che è possibile ottenere ottimi risultati, anche in termini di addestramento/ organizzazione del personale grazie all'ausilio della tecnologia digitale per le immagini e con un adeguato programma di istruzione, aggiornamento e monitoraggio del personale.

Ovviamente noi ci stiamo riferendo a uno studio monocentrico, che ha valutato un numero di pazienti limitato, ma speriamo che questa nostra esperienza preliminare possa essere punto di partenza per uno studio multicentrico e comparativo tra la tecnica $\mathrm{BH}$ e quella a scala di corda.

\section{Disclosures}

Financial support: No financial support was received for this submission.

Conflict of interest: The authors have no conflict of interest.

\section{Appendice 1}

\section{Protocollo di disinfezione}

1. Insegnare al paziente a eseguire sempre un accurato lavaggio sociale del braccio prima di ogni seduta dialitica. Questo ha lo scopo sia di ridurre la carica microbica che di coinvolgere attivamente il paziente.

2. Circa 15 minuti prima dell'ingresso in sala (e comunque mai oltre i 30 minuti), il paziente applica sulle croste una garza non sterile bagnata con soluzione fisiologica al fine di ammorbidire le croste e facilitarne la rimozione (Fig. 4.1).

3. L'infermiere indossa copricapo, mascherina ed esegue un accurato lavaggio delle mani. A questo punto indossa guanti monouso (non sono necessari guanti sterili).

4. Praticare la prima disinfezione con clorexidina al $2 \%$ su base alcolica, lasciando stazionare per almeno 30 secondi, come indicato dal produttore (Fig. 4.2).

5. Rimuovere le croste con tecnica asettica utilizzando l'apposito dispositivo "leva-crosta" sterile in dotazione con ogni ago smusso (15/16 Gauge, Nipro Medical Corporation): uno per ogni sito di puntura. Questa manovra richiede molta attenzione. Le croste vanno rimosse scollandole delicatamente intorno ai lembi fino a ottenerne la completa rimozione, esercitando il minor trauma possibile (Fig. 4.3). I maggiori problemi riscontrati in questo passaggio sono i seguenti:

- La crosta non si scolla completamente e rimane attaccata per un piccolo tratto alla cute marginale sana; in questo caso mai strappare la crosta verso la cute marginale sana ma procedere sempre dal lato opposto per evitare di allargare l'ingresso del tunnel cutaneo e quindi di incrementare il rischio infettivo.

- La crosta potrebbe frammentarsi; in questo caso rimuovere scrupolosamente ogni frammento perché potrebbe rappresentare un veicolo di infezione. 


\section{PROCEDURA DI DISINFEZIONE}

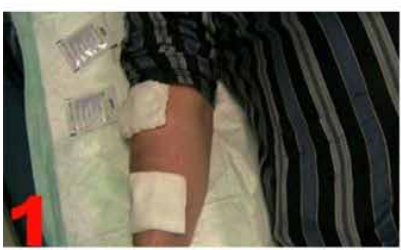

ammorbidire le croste

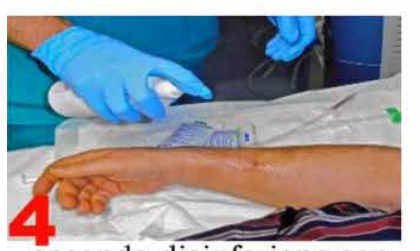

seconda disinfezione con clorexidina $2 \%$ su base alcolica o iodiopodivone 7,5 $\%$ su base acquosa

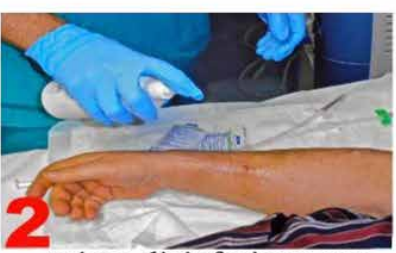

prima disinfezione con clorexidina $2 \%$ su base alcolica o iodiopodivone $7,5 \%$ su base acquosa

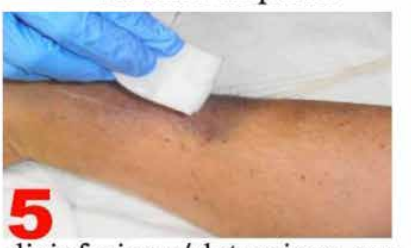

disinfezione/detersione con device sterile imbevuto di clorexidina $0,5 \%$ su base acquosa

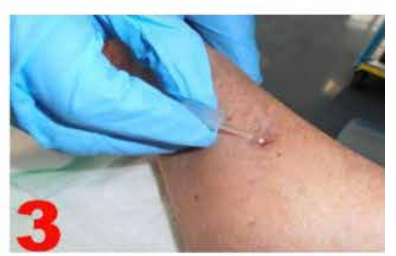

rimuovere con tecnica asettica la crosta con l'apposito device

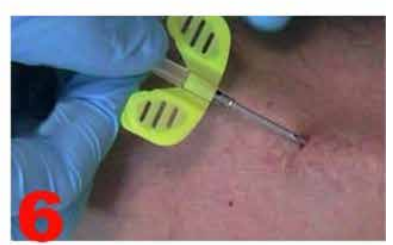

posizionare l'ago-fistola
Fig. 4 - Illustrazione delle fasi salienti della procedura di disinfezione utilizzata nel nostro centro.
6. Dopo aver rimosso completamente la crosta si esegue una seconda disinfezione con clorexidina $2 \%$ su base alcolica in forma spray (Fig. 4.4).

7. Realizzare una successiva ulteriore detersione della cute con una salviettina sterile imbevuta di clorexidina $0,5 \%$ su base acquosa, con l'obiettivo di rimuovere gli eventuali residui di croste e l'alcol in eccesso, che potrebbero determinare sensibilizzazione della cute con arrossamento (Fig. 4.5). Per evitare arrossamenti si dovrebbe sempre tener conto dei tempi di contatto specifici per ogni disinfettante. In caso di intolleranza alla clorexidina $2 \%$ su base alcolica si è utilizza la soluzione disinfettante con iodiopovidone su base acquosa $7,5 \%$, con tempo di contatto maggiore (circa 2 minuti).

8. Procedere al posizionamento degli aghi smussi (Fig. 4.6).

\section{Bibliografia}

1. Ethier J, Mendelssohn DC, Elder SJ, et al. Vascular access use and outcomes: an international perspective from the Dialysis Outcomes and Practice Patterns Study. Nephrol Dial Transplant. 2008;23(10):3219-26.

2. Howard AD, Howard RS, Goldstein SL, Meyer KB. Fistula First Breakthrough Initiative (FFBI): lessons about arteriovenous fistula prevalence goals. Am J Kidney Dis. 2013;61(3):523-5.

3. Nadeau-Fredette AC, Johnson DW. Con: Buttonhole cannulation of arteriovenous fistulae. Nephrol Dial Transplant. 2016;31(4):525-8.

4. Nesrallah GE. Pro: Buttonhole cannulation of arteriovenous fistulae. Nephrol Dial Transplant. 2016;31(4):520-3.

5. Doss S, Schiller B, Moran J. Buttonhole cannulation--an unexpected outcome. Nephrol Nurs J. 2008;35(4):417-9.

6. Twardowski ZJ, Kubara H. Different sites versus constant sites of needle insertion into arteriovenous fistulas for treatment by repeated dialysis. Dial Transplant. 1979;8:978-80.

7. Evans LM. Buttonhole cannulation for haemodialysis: a nursing review Renal Society of Australasia Journal. 2012;8(3):146-51.

8. van Loon MM, Goovaerts T, Kessels $A G H$, van der Sande FM, Tordoir JHM. Buttonhole needling of haemodialysis arteriovenous fistulae results in less complications and interventions compared to the rope-ladder technique. Nephrol
Dial Transplant. 2009;25:225-30.

9. Ludlow V. Buttonhole cannulation in hemodialysis: improved outcomes and increased expense-is it worth it? CANNT J. 2010;20:29-37.

10. Verhallen AM, Kooistra MP, van Jaarsveld BC. Cannulating in haemodialysis: rope-ladder or buttonhole technique? Nephrol Dial Transplant. 2007;22(9):2601-04.

11. Murcutt G. Buttonhole cannulation: Should this become the default technique for dialysis patients with native fistulas? Summary of the EDTNA/ERCA Journal Club discussion Autumn 2007. J Ren Care. 2008;34:101-08.

12. Nesrallah GE, Cuerden M, Wong JH, Pierratos A. Staphylococcus aureus bacteremia and buttonhole cannulation: long-term safety and efficacy of mupirocin prophylaxis. Clin J Am Soc Nephrol. 2010;5(6):1047-53,

13. Chow J, Rayment G, San Miguel S, Gilbert M. A randomised controlled trial of buttonhole cannulation for the prevention of fistula access complications. J Ren Care. 2011;37(2): 8593.

14. Ball LK. Improving Arteriovenous Fistula Cannulation Skills. Nephrol Nurs J. 2005;32(6):611-7.

15. Lombardi M. Il tallone d'Achille della moderna emodialisi. G Tec Nefrol Dial. 2002;14(Suppl. 3):3-5. 